\title{
Yes, Sexual Harassment Still Drives Women Out of Physics
}

\section{A survey of female undergraduates in physics found that three quarters of them experience some form of sexual harassment, leaving them alienated from the field.}

\section{by Julie Libarkin*}

Y

ou may be someone who thinks that sexual harassment is a "thing of the past" or that it's experienced by "only a few" women. These statements don't capture reality. As recently as 2014, a study found that sexual harassment affects the majority of women in science, technology, engineering, mathematics, and medicine (STEMM) [1]. Now, a comprehensive survey of female undergraduates in physics has uncovered a similarly disturbing situation for this group of women at the start of their careers. Lauren Aycock, of the University of Maryland, College Park, and the National Institute of Standards and

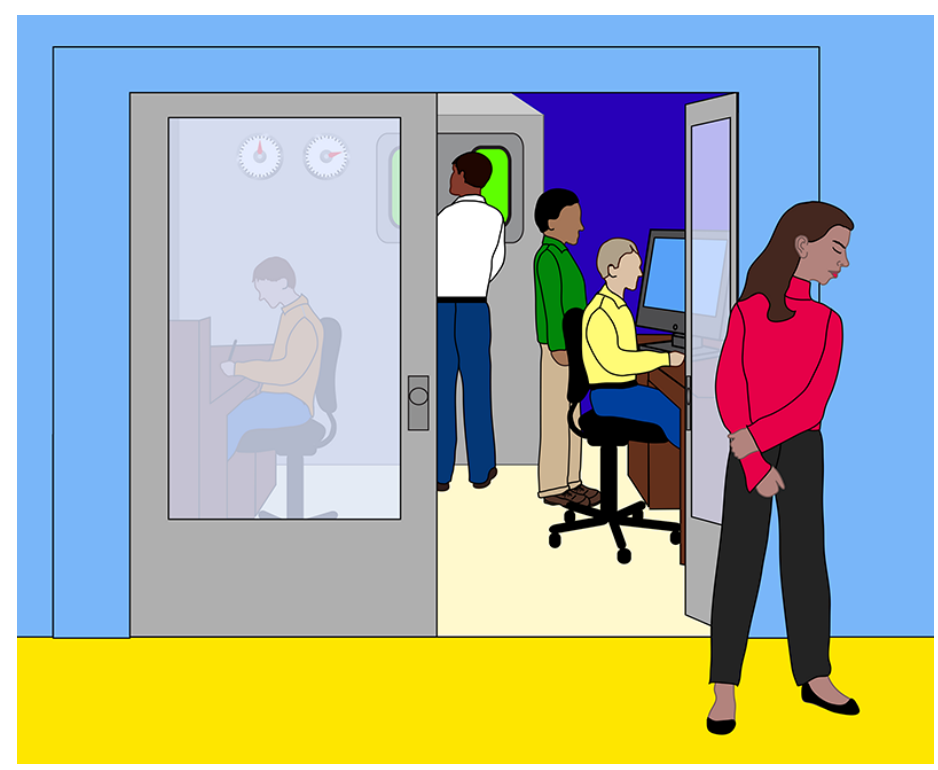

Figure 1: A survey of female physics undergraduates in the US found that experiencing sexual harassment can create a sense of isolation and diminish confidence in ability [2]. (Joan Tycko/APS)

\footnotetext{
* Department of Earth and Environmental Sciences, Michigan State University, Michigan, USA
}

Technology, Maryland, and colleagues surveyed female attendees at the 2017 Conferences for Undergraduate Women in Physics (CUWiP) [2]. They found that nearly threequarters of the roughly 500 respondents had experienced some form of sexual harassment and that these experiences correlated with a sense of not belonging to the field. This finding won't surprise most women in STEMM, but it may shock men in STEMM, who are often unaware of sexual harassment's pervasiveness and damage.

Broadly defined, sexual harassment is unwelcome or inappropriate behavior of a sexual nature that creates an uncomfortable or hostile environment. It comes in various forms, both subtle and overt, but three specific types were considered in the Aycock et al. study. "Sexist gender harassment" describes hostile or insulting remarks and actions based on one's gender, such as saying women cannot do physics. By contrast, "sexual gender harassment" refers to sexual remarks or conduct, like commenting on the shape of a woman's body. A third form of sexual harassment is unwanted sexual attention, such as requests for sexual favors or unwanted touching.

Unfortunately, these behaviors are entrenched within societies around the world, and they persist because of permissive institutions, inadequate reporting mechanisms, and the normalization of sexual violence [3]. To change this culture, we need to first assess where we are. Studies of international academic environments suggest that sexual harassment is common [3-5], with as many as 70\% of women reporting sexual harassment experiences. Students are most likely to experience harassment by their peers, but the perpetrators can also be faculty and staff [6]. Sexual harassment is often pervasive and continuous: most victims have more than one experience and from more than one person [3].

Despite this extensive scholarship, many people are unaware of the toll sexual harassment takes on its victims. Experiencing sexual harassment increases a woman's likelihood of leaving a STEMM career [3]. And for those women who do stick with their field, harassment hurts their career, economic standing, and well-being [7]. In short, unchecked harassment creates a drain on talent through lost work, lost ideas, and lost people [3].

Understanding the extent and effect of sexual harassment 
for physics undergraduates is essential because this is the first stage of becoming a physicist. CUWiP are a collection of simultaneous regional meetings run by the American Physical Society (the publisher of Physics) to encourage women to stay in physics. In their study, Aycock et al. collected online surveys from 464 female CUWiP attendees-roughly 5-10\% of the female undergraduate physics majors in the US at the time [2]. The students were asked if they had experienced sexist gender harassment, sexual gender harassment, or unwanted sexual attention "in the context of physics"-such as in a physics lab, classroom, or at a physics department event. The researchers then analyzed the relationship between these experiences and two variables often correlated with career persistence. One is a "sense of belonging," the extent to which one feels connectedness to or identification with a given community. The other is the "imposter phenomenon," where one largely attributes his or her success to luck, hard work, or preferential treatment rather than ability.

Aycock et al. found that experiencing sexual harassment correlated to a negative sense of belonging and an exacerbated sense of the imposter phenomenon. Three-quarters $(74.3 \%)$ of respondents experienced at least one form of sexual harassment; nearly half $(47.9 \%)$ experienced multiple forms. Of the women experiencing harassment, $91.3 \%$ experienced sexist gender harassment, $34.2 \%$ experienced sexual gender harassment, and $32.6 \%$ experienced unwanted sexual attention. The findings align with a 2016 study that demonstrated the prevalence of sexual harassment of graduate women in physics [8]. Using regression models, Aycock et al. found that only sexist gender harassment was associated with a deteriorated sense of belonging. However, respondents were more likely to attribute their success to hard work, luck, or external perceptions-instead of ability-if they had experienced either sexist or sexual gender harassment.

Does sexual harassment inhibit women from choosing physics as a long-term career, as these results suggest? That's a question worth asking after decades of effort to bring more women into physics has had little traction: only about $20 \%$ of physics degrees at all levels go to women. Aycock et al. recognize that surveys provide only one step towards assessing the damage from sexual harassment and that further study is needed to unpack their findings. For example, we would learn more by asking women who have left physics why they did so.

Studies such as this one are a wake-up call to all members of our community to advocate for change. An effective step can be as simple as calling out problematic behavior. For example, the \#MeToo movement on social media-set in motion by activist Tarana Burke in 2006-has been adopted by academics to call out sexual harassment (\#AcademiaToo). When bystanders confront a harasser, victims are often em- powered to do the same. Considering that workplaces with high incidences of sexual harassment tend to also be uncivil and disrespectful [9], addressing sexual misconduct may therefore improve the culture for everyone.

When sexual harassment goes unchecked, physics loses great people, great minds, and great potential. It's worth noting that sexual harassment disproportionately affects people of color, people with disabilities, and members of the LGBTQ+ community [3]. This may partially explain why the physics community has long been male dominated, and is disproportionately able-bodied and white [10]. It's up to all of us to see the culture as it truly is, acknowledge sexual harassment as an ingrained problem, and take steps to ensure that all physicists have a welcoming and respectful place to work.

This research is published in Physical Review Physics Education Research.

\section{REFERENCES}

[1] K. B. H. Clancy, R. G. Nelson, J. N. Rutherford, and K. Hinde, "Survey of academic field experiences (SAFE): Trainees report harassment and assault," PLoS ONE 9, e102172 (2014).

[2] L. M. Aycock, Z. Hazari, E. Brewe, K. B. H Clancy, T. Hodapp, and R. M. Goertzen, "Sexual harassment reported by undergraduate female physicists," Phys. Rev. Phys. Educ. Res. 15, 010121 (2019).

[3] Sexual harassment of women: Climate, culture, and consequences in academic sciences, engineering, and medicine, edited by P. A. Johnson, S. E. Widnall, and F. F. Benya (The National Academies Press, Washington, DC, 2018).

[4] Change the course: National report on sexual assault and se xual harassment at australian universities (Australian Human Rights Commission, Sydney, 2017).

[5] Power in the academy: Staff sexual misconduct in UK higher education (National University of Students, London, 2017).

[6] M. N. Rosenthal, A. M. Smidt, and J. J. Freyd, "Still Second Class: Sexual harassment of graduate students," Psychol. Women Q. 40, 364 (2016).

[7] L. Howe-Walsh and S. Turnbull, "Barriers to women leaders in academia: Tales from science and technology," Stud. High. Educ. 41, 415 (2014).

[8] R. S. Barthelemy, M. McCormick, and C. Henderson, "Gender discrimination in physics and astronomy: Graduate student experiences of sexism and gender microaggressions," Phys. Rev. Phys. Educ. Res. 12, 020119 (2016).

[9] S. Lim and L. M. Cortina, "Interpersonal Mistreatment in the Workplace: The Interface and Impact of General Incivility and Sexual Harassment," J. Appl. Psychol. 90, 483 (2005).

[10] What does a physicist look like? (Institute of Physics, London, 2016).

10.1103/Physics.12.43 